\title{
Experimental Digital Animation in Fluid Dynamics
}

\author{
Irina Znamenskaya ${ }^{1}$, Nikolay Sysoev ${ }^{1}$ and Igor Doroshchenko ${ }^{1}$ \\ ${ }^{1}$ Faculty of Physics, Lomonosov Moscow State University, 1-2 Leninskiye Gory, Moscow, 119991, Russia
}

\begin{abstract}
Digital imaging became one of the main tools for studying unsteady flows. Modern high-speed cameras support video recording at high frame rates which makes it possible to study extended high-speed processes. We demonstrate here different animations: water temperature field evolution with a frame rate of $115 \mathrm{~Hz}$; high-speed shadowgraph visualisation of different flows - water jet formation process (100 000 frames / s), shadowgraph animations of the shock waves created by the pulsed discharges (124 000 frames / s). Also, as an example of plasma flow visualization technique, we offer 9 sequential images of the shock wave - pulse gas discharge visualization obtained by the high-speed CCD camera with the 100 ns delay between frames. We developed in-house software based on the machine vision and learning techniques for automatic flow animations processing. The examples of the automatic oblique shock detection using Canny edge detection and Hough transform and thermal plume detection based on the pre-trained convolutional neural network are provided and discussed.
\end{abstract}

\section{Keywords}

Flow visualization, thermographic animation, high-speed shadowgraph animation, discharge flow visualization, digital image processing, convolutional neural networks, edge detection, Hough transform.

\section{Introduction}

Non-stationary processes in gas, liquids, plasmas, multiphase media were recorded using equipment, respectively beginning from direct observation with the eyes, then observation using pinhole cameras, glass optics [1,2]. From the middle of the 19th century, photographic equipment began to be used for scientific visualization. French naturalist J. E. Marey was the first to use multi-frame photography to record flow movements. His chronophotographic gun (1882) was capable to record 12 consecutive frames per second. In the wind tunnel he visualized flow around obstacles and the transition to turbulence using a smoke system. Later film cameras, video cameras, and high-speed drum cameras were used. In the middle of the 20th century, electro-optical devices appeared. Modern digital highspeed cameras can record fast processes in fluids at rate up to 1 million frames / s.

One of the aims of gaining visual information about fluid dynamic processes is the creation of experimental databases for verification of CFD software. Actual problem of the CFD codes verification is matching of experimental and calculated data - 2D flow images.

Visualization equipment while capturing films of non-stationary flows should have exposure time less then minimal flow characteristic time; thus is the time interval between 2 frames.

GraphiCon 2021: 31st International Conference on Computer Graphics and Vision, September 27-30, 2021, Nizhny Novgorod, Russia EMAIL: znamen@phys.msu.ru (I. Znamenskaya);nn.sysoev@physics.msu.ru (N. Sysoev);

doroshenko.igor@physics.msu.ru (I. Doroshchenko)

ORCID: 0000-0001-6362-9496 (I. Znamenskaya); N. Sysoev; 0000-0002-0488-0020 (I. Doroshchenko)

(c) (i) (C) 2021 Copyright for this paper by its authors.

Use permitted under Creative Commons License Attribution 4.0 International (CC BY 4.0).

CEUR Workshop Proceedings (CEUR-WS.org) 


\section{Thermographic animation}

Modern thermal imaging cameras resolution is considerably lower than that of optical cameras. The frame rate is less than 1000 frames / s. Figure 1 presents subsequent thermographic images of turbulent boundary water flow at $115 \mathrm{~Hz}$ - submerged impact jet (false colors). Being registered through an IRtransparent window, thermal imager visualizes IR radiation from a thin near-surface layer $[3,4]$. The time dependences of the measured thermal signals at each point of animation are extracted using FLIR software (Figure 2).
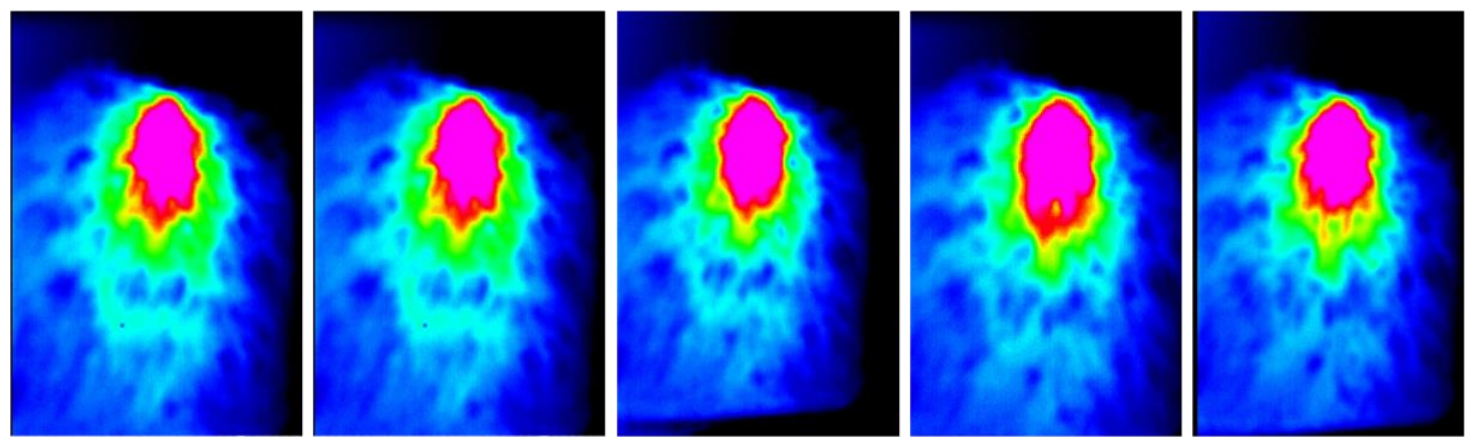

Figure 1: Thermographic recording at $115 \mathrm{~Hz}$
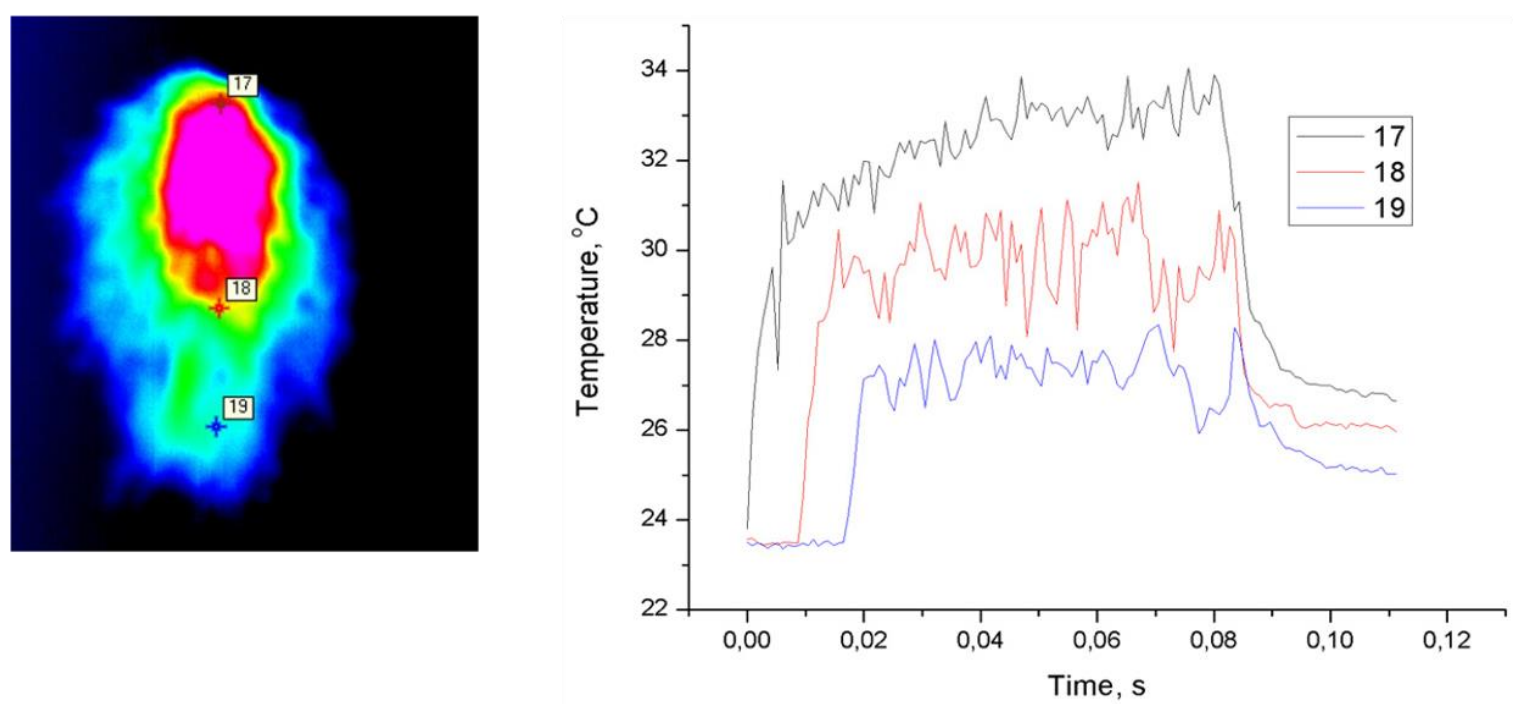

Figure 2: Flow pulsations in 3 different points

\section{High-speed shadowgraph imaging}

The usage of modern optical high-speed digital cameras has made it possible to study high-speed non-stationary processes with a shooting frequency of more than a million frames per second. Figure 3 shows 5 shadowgraph frames of the water jet formation process: visualization at the top area motion. The leader speed (measured manually) increases from 30 to $250 \mathrm{~m} / \mathrm{s}$. Time interval between frames $0.03 \mathrm{~ms}$ is enough for speed measurement. 

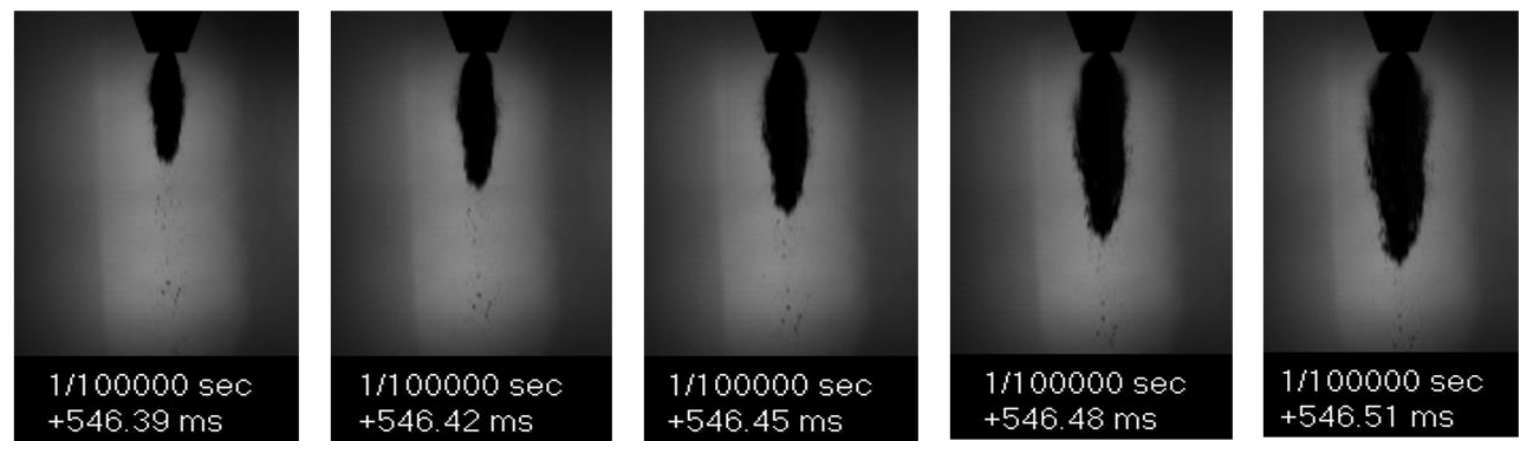

Figure 3: Shadowgraph images captured by high-speed camera (light absorption) 100000 frames / s

Figure 4 shows 4 sequential shadowgraph frames of the motion of 2 shock waves initiated by a nanosecond discharges when shooting with a high-speed digital camera (124 000 frames/s). Cylindrical shock (blast) wave interacts with plane shock.
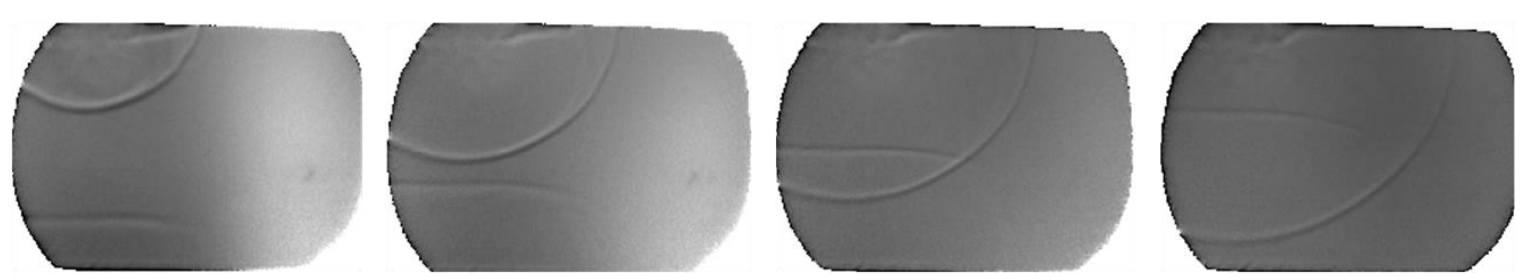

Figure 4: Shadowgraph images (light refraction) captured by high-speed camera at 124000 frames/s

Supersonic gas flow structure may be visualized by the electrical discharge [5]. Discharge plasma in gas volume follows shapes of discontinuities, inhomogeneities, streamlines because discharge electric current strongly depends on the $\mathrm{E} / \mathrm{N}$ ratio [5, 6]. Figure 5 presents 9 frames of discharge glow zone: ionization upon interaction of a shock wave with a pulse volume discharge in the nanosecond range. Images are taken with high-speed CCD camera connected to PC. Animation of 9 frames is assembled to study the dynamics of nanosecond-lasting plasma configuration evolution.

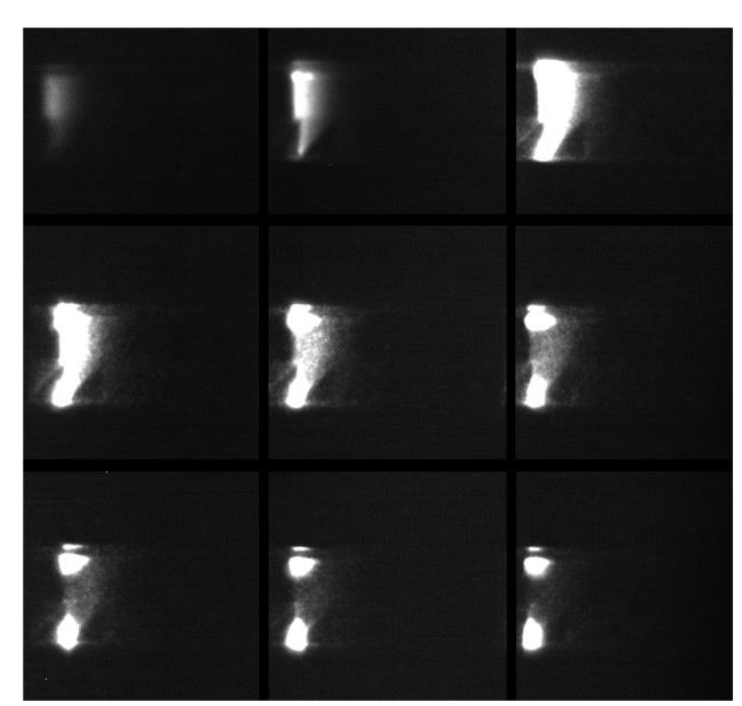

Figure 5: Images of discharge glow captured by high-speed camera; 100 ns exposure; 100 ns time interval between frames.

\section{Computer vision and machine learning}

The actual problem of a panoramic experiment data analysis is the problem of big data. In the experiments today a huge amount of digital information is accumulated, obtained during video filming 
with digital cameras, thermal imagers, etc. The development of digital technologies leads to a multiple increase in the data array of the parameters of thermophysical flow fields; large arrays of digital data appear which do not make it possible to process it manually. Thus, modern video films recording the evolution of turbulent fluid flows based on shadow methods, tracing, thermography require processing and qualified analysis. The transition to another level of data analysis is inevitable.

A huge amount of digital information is accumulated in the experiments, obtained during video filming with digital cameras, thermal imagers, etc. When working with big data, machine learning may help analyzing large data arrays (in our case, flow images) [7]. So far, very few papers have been devoted to this problem, but their number is growing rapidly. Neural networks can effectively capture gas flow structures on large datasets [8], predict [9] and reconstruct [10] flow development using Image Retrieval, Template Matching, Parameters Regression, Spatiotemporal Prediction and other techniques. Deep learning may be used to model high-dimensional gas-dynamic systems such as turbulence [11]. Image classification and object detection systems are being developed, for example, for shock wave detection [8], bow-shock refraction angle tracking [12] or vortex wakes detection and classification [13].

Different computer vision algorithms are also being used for processing of digital animations of flows. The most used methods are edge detection, background image subtraction, noise removal [14].

In the present study we made two software for automatic flow structures detection and tracking. The first one is our in-house code for shock wave detection based on the modified Canny edge detection and Hough transform algorithms $[15,16]$. Edge detection is used to represent possible shock wave boundaries and the Hough transform is used to find boundaries close to the straight line. Also, we apply some line length and angle filters in our code and combining close short lines into one. Figure 6 shows an example of shadowgraph image processing using our software: oblique shock detection and automatic angle calculation. The entire video contains several hundred frames. The oblique shock was created by a small obstacle placed on the bottom wall of the shock tube channel.
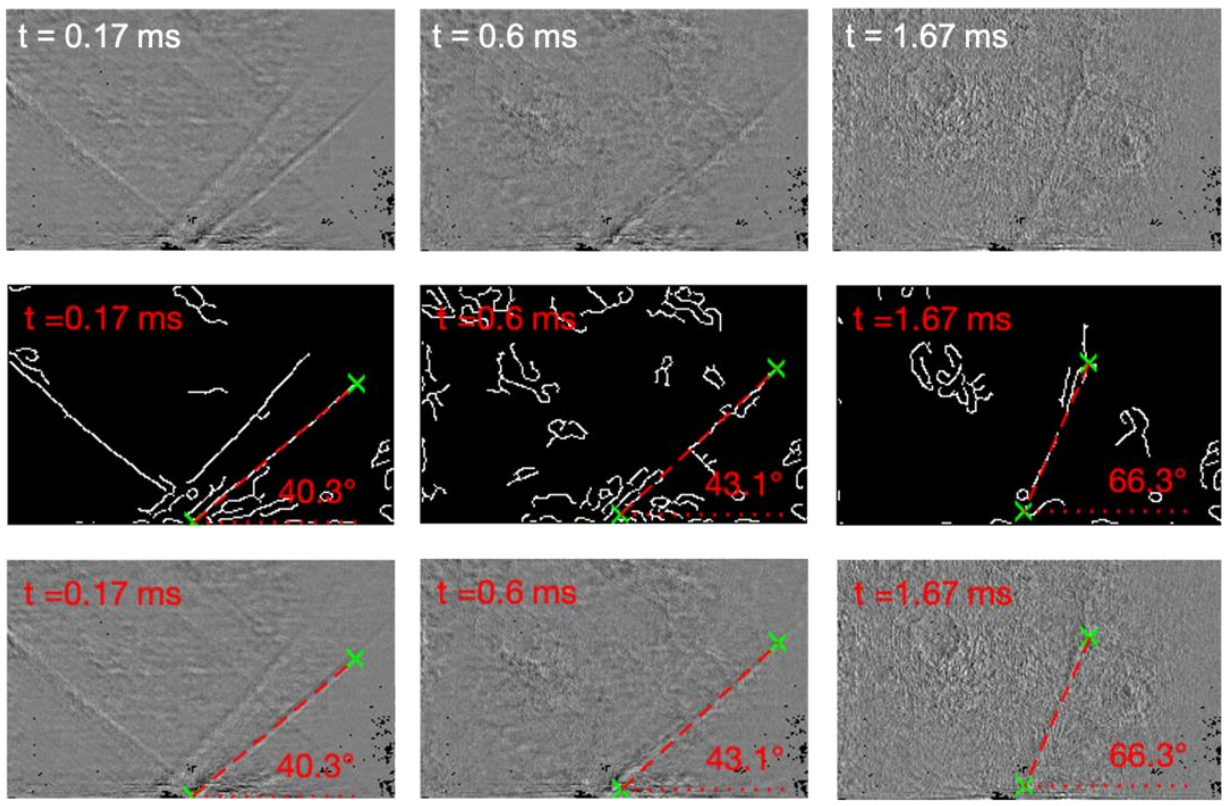

Figure 6: Automatic image processing: applying edge detection and Hough transform to detect oblique shock and calculate its angle; first row - source images; second row - edge and line detection; third row - source image with the detected oblique shock and calculated angle.

The second software was developed using the convolutional neural network based on the wellknown YOLOv2 architecture. The network was trained to detect three classes of objects on the images: shock waves, convective plumes, and tracer particles. We used up to 800 images for training. Some of them are featured in our online gallery [17]. The detailed information about the software is given in $[15,16]$. Figure 7 shows the example frames of the post-discharge thermal plume development and its automatic detection by the neural network. The full animation contains several thousand images. 

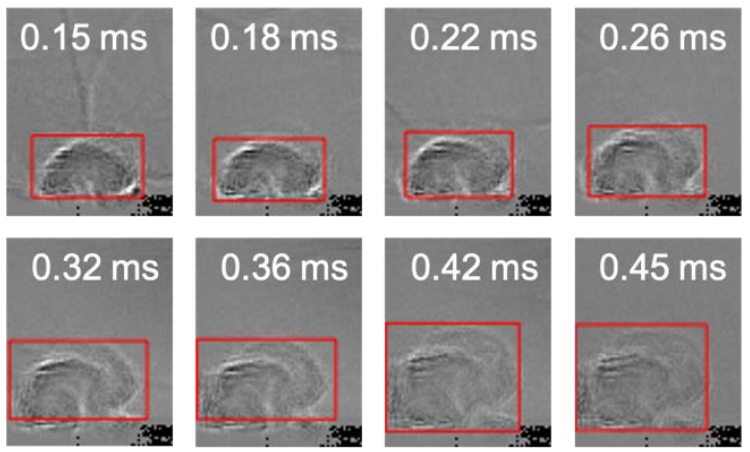

Figure 7: Automatic image processing: thermal plume detection using convolutional neural network.

\section{Conclusion}

Digital scientific animation is one of the main tools for studying non-stationary flows. Modern highspeed cameras support video recording at a frame rate of up to 1000000 frames / s which makes it possible to study high-speed processes. Digital animations may match CFD calculations to validate the CFD code and improve its accuracy. We presented thermographic animations obtained at $115 \mathrm{~Hz}$. to measure fluid temperature evolution with a high frame rate in different image points. Also, we provide high-speed shadowgraph images of different flows. Water jet formation process was recorded at a frame rate of 100000 frames / s (example of light absorption). Shadowgraph animations of the shock waves created by the pulsed discharges were recorded at 124000 frames / s (light refraction example). Also, we present 9 sequential images obtained by the special high-speed CCD camera with the 100 ns delay between frames. Such short time intervals are suitable for pulsed electrical discharge visualization, it can be presented as short animation as well.

Also, for big visual animated data we developed in-house software based on the machine vision and learning techniques for automatic animations processing. The examples of the automatic oblique shock detection using Canny edge detection and Hough transform and thermal plume detection based on the pre-trained convolutional neural network were provided.

\section{Acknowledgements}

This study was carried out within the framework of the Development Program of the Interdisciplinary Scientific and Educational School of Moscow State University "Photonic and Quantum Technologies: Digital Medicine.”

\section{References}

[1] V. N. Emelyanov, K. N. Volkov, Visualization of physical and mathematical modeling data in gas dynamics, Moscow: Fizmatlit, 360 p., 2018, ISBN: 978-5-9221-1774-6.

[2] G. S. Settles, Schlieren and Shadowgraph Techniques: Visualizing Phenomena in Transparent Media, Springer, 2001. ISBN: 978-3-642-56640-0.

[3] I. A. Znamenskaya, E. Yu. Koroteeva, Ya. N. Shirshov, A. M. Novinskaya, N. N. Sysoev, High speed imaging of a supersonic waterjet flow, Quantitative InfraRed Thermography Journal 142 (2017) 185-192. doi: 10.1080/17686733.2016.1243749.

[4] M. A. Bolshukhin, I. A. Znamenskaya, V. I. Fomichev, A method of quantitative analysis of rapid thermal processes through vessel walls under nonisothermal liquid flow, Dokl. Phys. 60 (2015) 524-527. doi: 10.1134/S1028335815110014.

[5] M. Nishio, S. Sezaki, H. Nakamura, Visualization of flow structure around a hypersonic re-entry capsule using the electrical discharge method, Journal of Visualization 7 (2004) 151-158. doi: 10.1007/BF03181588.

[6] I. A. Znamenskaya, D. A. Koroteev, N. A. Popov, A nanosecond high-current discharge in a supersonic gas flow, High Temperature 43 (2005) 817-824. doi: 10.1007/s10740-005-0129-x. 
[7] S. L. Brunton, B. R. Noack, P. Koumoutsakos, Machine Learning for Fluid Mechanics, Annual Review of Fluid Mechanics, 52 (2020) 477-508. doi: 10.1146/annurev-fluid-010719-060214.

[8] M. Monfort, T. Luciani, J. Komperda, B. Ziebart, F. Mashayek, G. E. Marai, A Deep Learning Approach to Identifying Shock Locations in Turbulent Combustion Tensor Fields, Modeling, Analysis, and Visualization of Anisotropy (2017) 375-392. doi: 10.1007/978-3-319-61358-1_16.

[9] R. Harel, M. Rusanovsky, Y. Fridman, A. Shimony and G. Oren, Complete Deep Computer-Vision Methodology for Investigating Hydrodynamic Instabilities, In: Jagode H., Anzt H., Juckeland G., Ltaief H. (eds) High Performance Computing. ISC High Performance 2020. Lecture Notes in Computer Science, vol. 12321, 2020. pp. 61-80. doi: 10.1007/978-3-030-59851-8_5.

[10] C. Ott, C. Pivot, P. Dubois, Q. Gallas, J. Delva, M. Lippert and L. Keirsbulck, Pulsed jet phaseaveraged flow field estimation based on neural network approach, Experiments in Fluids 6279 (2021). doi: 10.1007/s00348-021-03180-0.

[11] J. Kutz, Deep learning in fluid dynamics, Journal of Fluid Mechanics 814 (2017) 1-4. doi: $10.1017 / \mathrm{jfm} .2016 .803$.

[12] M. Dehghan Manshadi, H. Vahdat-Nejad, M. Kazemi-Esfeh and M. Alavi, Speed Detection in Wind-tunnels by Processing Schlieren Images, IJE TRANSACTIONS A: Basics 297 (2016) 962967. doi: 10.5829/idosi.ije.2016.29.07a.11.

[13] B. Colvert, M. Alsalman, E. Kanso, Classifying vortex wakes using neural networks, Bioinspiration \& Biomimetics, 132 (2018). doi: 10.1088/1748-3190/aaa787.

[14] G. Li, M. Burak Agir, K. Kontis, K. Ukai and S. Rengarajan, Image Processing Techniques for Shock Wave Detection and Tracking in High Speed Schlieren and Shadowgraph Systems, Journal of Physics: Conference Series 1215 (2019). doi: 10.1088/1742-6596/1215/1/012021.

[15] I. A. Znamenskaya, I. A. Doroshchenko, Edge detection and machine learning for automatic flow structures detection and tracking on schlieren and shadowgraph images, Journal of Flow Visualization and Image Processing $28 \quad 4 \quad$ (2021) 1-26. doi: 10.1615/JFlowVisImageProc.2021037690.

[16] I. Znamenskaya, I. Doroshchenko, D. Tatarenkova, Edge Detection and Machine Learning Approach to Identify Flow Structures on Schlieren and Shadowgraph Images, CEUR Workshop Proceedings 2744 (2020) 1-14. doi: 10.51130/graphicon-2020-2-3-15.

[17] Gallery of Photos and Videos, 2021. URL: http://molphys.phys.msu.ru/galery. 